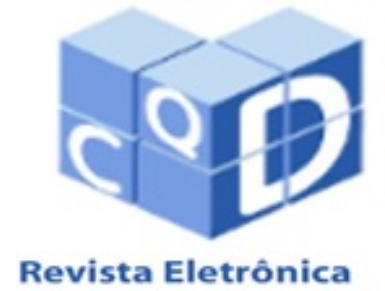

Paulista de Matemática

ISSN 2316-9664

Volume 7, dez. 2016

Edição ERMAC

Gabriela Colovati de Almeida

Universidade Estadual

Paulista/UNESP Botucatu

gabriela_cda@ibb.unesp.br

Fernando Luiz Pio dos Santos

Universidade Estadual

Paulista/UNESP Botucatu

flpio@ibb.unesp.br

\section{Modelo matemático espaço-discreto para análise de propagação da dengue}

Mathematical discrete-space model for analysis of the spread of dengue fever

\section{Resumo}

Neste trabalho, a dinâmica de transmissão da dengue foi analisada por intermédio de simulações numéricas do sistema de equações diferenciais ordinárias não-lineares, considerando a mobilidade humana e a transmissão vertical como fatores de interesse para descrever o persistência e difusão dessa enfermidade. O sistema de equações é composto pela interação dos compartimentos da população de humanos e vetores, além do compartimento da fase aquática para o caso de dois patches. O método numérico utilizado para a resolução do sistema foi o método de Runge-Kutta de quarta ordem. Examinando os resultados obtidos, temos que o patch que estava livre da doença inicialmente, foi mais afetado pela transmissão vertical do que pela mobilidade humana. Dessa forma, podemos sugerir o reforço das ações de controle usadas na fase aquática para reprimir a transmissão da dengue.

Palavras-chave: Biomatemática. Equações diferenciais ordinárias. Mobilidade humana. Patches.

\begin{abstract}
In this work, the dynamics of dengue fever transmission was analyzed through numerical simulations of the system of non-linear ordinary differential equations, considering human mobility and vertical transmission as factors of interest to describe the persistence and diffusion of this disease. The system of equations consists of the interaction of the human population compartments and vectors, as well as the aquatic phase compartment for the case of two patches. The numerical method used to solve the system was the fourth-order Runge-Kutta method. Examining the results obtained, the patch that was free of the disease initially was more affected by vertical transmission than by human mobility. Thus, we can suggest the reinforcement of control actions used in the aquatic phase to suppress the transmission of dengue.
\end{abstract}

Keywords: Biomathematics. Ordinary differential equations. Human mobility. Patches. 


\section{Introdução}

Um dos graves problemas de saúde pública mundial, ocorrendo em territórios tropicais e subtropicais, é a proliferação da doença infecciosa dengue (DENGUE, 2009). Como os seres humanos são os hospedeiros da doença, consideramos aqui a mobilidade humana como um fator de extrema importância na propagação dessa enfermidade. Segundo Stolerman; Coombs; Boatto (2015), em uma área urbana, a propagação da dengue se dá possivelmente pelo movimento humano de todos os dias, em que esses se movem por motivos distintos desempenhando um papel fundamental na distribuição espacial.

Consideramos também, a transmissão vertical que ocorre na fase aquática, podendo ser transovariana ou transovo, como um evento que causa alterações na biologia do vetor, ocorrendo assim, mudanças na disseminação e permanência da doença (LEANDRO, 2015).

As equações diferenciais são ferramentas matemáticas de extrema importância para descrever fenômenos reais. Estas equações são modelos físicos úteis para a compreensão de situações ou problemas reais. Para Bassanezi (2011), a modelagem matemática de situações reais pode ser facilitada quando se usa sistemas interligados, chamados de compartimentos. Cada compartimento é determinado por suas propriedades físicas, trocando entre si e com o meio ambiente, materiais.

Uma abordagem clássica compartimental para a análise da propagação de doenças infecciosas foi estudada inicialmente por Kermack e McKendrick (1927), em que eles propuseram um modelo que dividia o tamanho total de uma população em três subpopulações. Assim, quando temos um agente patogênico que é ativo, podemos dividir a população de humanos em compartimentos distintos de acordo com a situação epidemiológica (ESTEVA; VARGAS, 1998; ESTEVA; YANG, 2015).

Podemos observar o crescente estudo de modelos matemáticos que trabalham com regiões bem definidas e estruturadas para descrever dinâmicas populacionais e principalmente a expansão de uma doença (ARINO; VAN DEN DRIESSCHE, 2003; BOWONG; DUMONT e TEWA, 2013; PUANGSUN; PATANARAPEELERT, 2012).

De acordo com Generali (2010), a dinâmica de um modelo metapopulacional trabalha com uma população dividida em subpopulações. Estas são locais e divididas em fragmentos, chamados de sítios ou patches (espaços discretos bem definidos). Os movimentos migratórios conectam os patches uns aos outros. Dessa forma, para examinar a dinâmica de transmissão da dengue, estendemos o modelo proposto por Newton e Reiter (1992) para ser aplicado na situação onde temos a existência desses espaços discretos.

Nesta abordagem são consideradas as interações entre as populações de humanos e mosquitos, um compartimento aquático com diferentes capacidades de suporte, e ainda, a circulação da doença dengue para um único sorotipo (ANDRAUD et al., 2012; BOWONG; DUMONT e TEWA, 2013; SANTOS, 2015). O objetivo foi efetuar simulações numéricas do novo modelo para o caso particular de dois patches, resolvendo-o pelo método de Runge-Kutta de quarta ordem, para investigar o efeito da interação humana de um patch que têm condições iniciais para a transmissão da dengue e de outro patch que está livre da doença. Na próxima seção enunciaremos o modelo matemático para dois patches.

\section{Modelo Matemático para dois Patches}

Alguns estudos utilizam a aplicação do modelo proposto inicialmente por Newton e Reiter

ALMEIDA, G. C.; SANTOS, F. L. P. Modelo matemático espaço-discreto para análise de propagação da dengue. C. Q. D. - Revista Eletrônica Paulista de Matemática , Bauru, v. 7, p. 78-87, dez. 2016. Ediçao ERMAC.

DOI: 10.21167/cqdvol7ermac201623169664gcaflps7887 - Disponível em: http://www.fc.unesp.br/\#!/departamentos/matematica/revista-cqd/ 
(1992) sem modificações (CIRINO; SILVA, 2004; GERHARDT, 2004). Entretanto, o presente trabalho ampliou o referido modelo, conferindo-lhe alterações, gerando o sistema estendido especialmente para dois patches, em que é constituído pelas equações diferenciais representando a fase aquática (ovos, larvas e pupas) com o parâmetro de proporção dos vetores que se tornam infecciosos ao nascerem (transmissão vertical) seguido das equações para as populações de humanos e vetores.

As variáveis de estado do modelo no tempo $t$ estão na Tabela 1. Os parâmetros biológicos do sistema (1) são dados pela Tabela 2 e foram retirados de Cirino e Silva (2004) e Thomé; Yang e Esteva (2010).

Tabela 1: Variáveis de Estado do Modelo.

\begin{tabular}{cc}
\hline Variável & Descrição da Variável \\
\hline$A$ & Fase aquática \\
$S_{h}$ & Densidade de humanos suscetíveis \\
$E_{h}$ & Densidade de humanos expostos \\
$I_{h}$ & Densidade de humanos infecciosos \\
$R_{h}$ & Densidade de humanos recuperados \\
$S_{v}$ & Densidade de vetores suscetíveis \\
$E_{v}$ & Densidade de vetores expostos \\
$I_{v}$ & Densidade de vetores infecciosos \\
\hline
\end{tabular}

Tabela 2: Parâmetros Biológicos do Modelo.

\begin{tabular}{c|c|c}
\hline Parâmetros & Descrição do Parâmetro & Valor \\
\hline$k$ & Fração de ovos viáveis $(0<k<1)$ & 0.8 \\
$\phi$ & Taxa de oviposição intrínsica $(0<\phi<1)$ & 0.9 \\
$\alpha$ & Proporção de vetores que se tornam & \\
& suscetíveis ao nascerem $(0<\alpha<1)$ & 0.5 \\
$\beta$ & Proporção de vetores que se tornam infecciosos & \\
& ao nascerem devido a transmissão vertical $(0<\beta<1)$ & {$[0-0.3]$} \\
$\mu_{A}$ & Taxa de mortalidade da fase aquática $\left(0<\mu_{A}<1\right)$ & 0.0583 \\
$M_{h}$ & Período da expectativa de vida dos humanos (dias) & 25000 \\
$D_{h}$ & Período latente intrínseco dos humanos (dias) & 5 \\
$P_{h}$ & Período de duração da infecção (dias) & 3 \\
$M_{v}$ & Período da expectativa de vida dos vetores (dias) & 4 \\
$D_{v}$ & Período latente extrínseco dos vetores (dias) & 10 \\
$c_{v h}$ & Taxa de transmissão da interação vetor-humano (dias $\left.{ }^{-1}\right)$ & 0.75 \\
$c_{h v}$ & Taxa de transmissão da interação humano-vetor (dias $\left.{ }^{-1}\right)$ & 0.375 \\
\hline
\end{tabular}

Portanto, o modelo proposto é dado pelo seguinte sistema (1), considerando dois patches: 


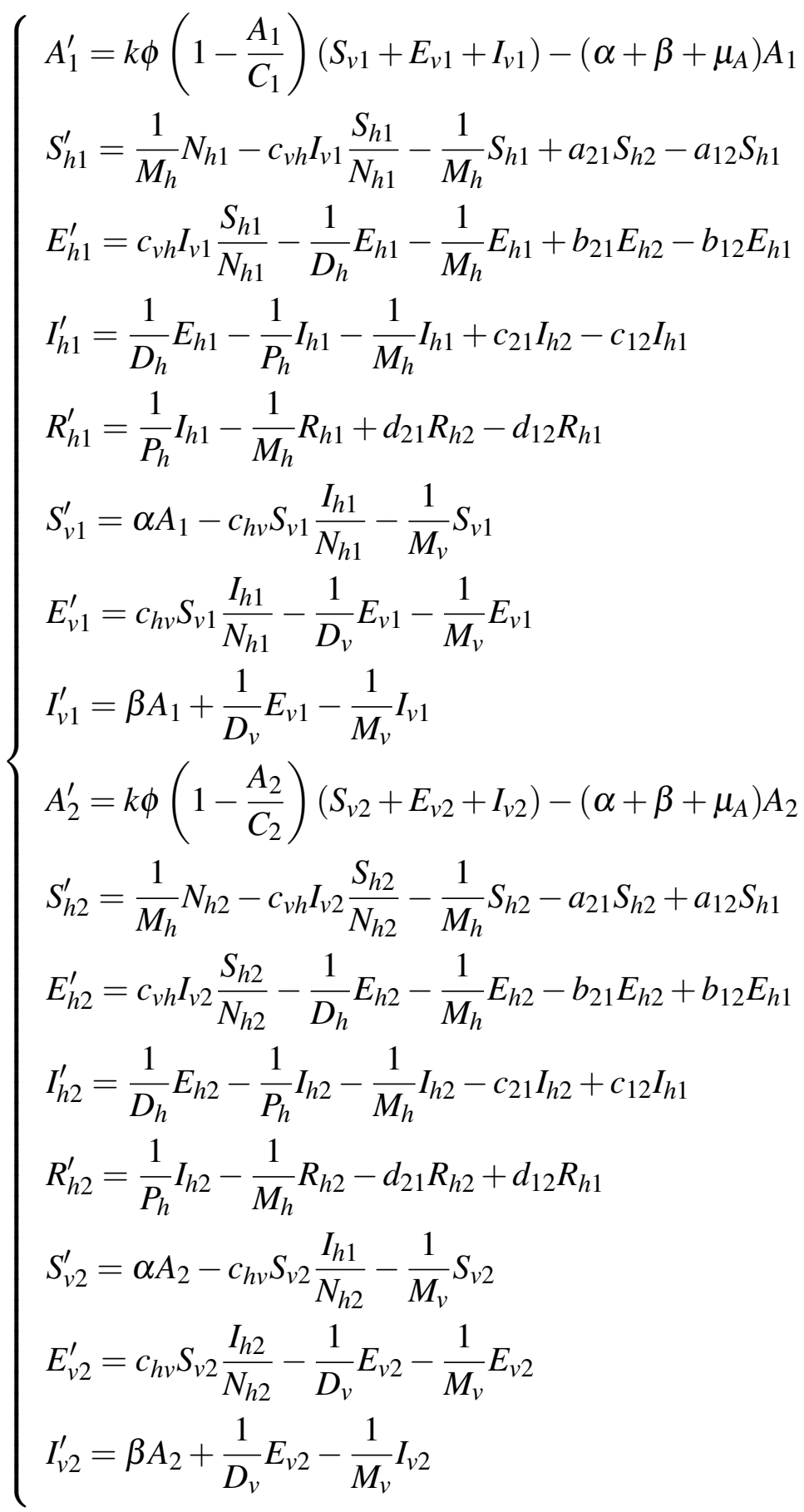

sendo $N_{h i}=S_{h i}+E_{h i}+I_{h i}+R_{h i}, C_{i}>0$ e $\left(1-\frac{A_{i}}{C_{i}}\right)$ a densidade total de humanos, a capacidade de suporte ambiental (espaços disponíveis e nutrientes) e o crescimento logístico de vetores, respectivamente, para os patches $i=1,2$. As taxas de movimentação humana são entre os dois patches são dadas por: $a_{12}, a_{21}, b_{12}, b_{21}, c_{12}, c_{21}, d_{12}$ e $d_{21}$.

A Figura 1 representa o fluxo de humanos entre os compartimentos . A seção seguinte descreve os resultados numéricos obtidos pela simulação numérica do modelo matemático que considera duas regiões distintas do espaço, isto é, dois patches. Para isto, estabelecemos cenários com o intuito de simular os efeitos da mobilidade humana, bem como os efeitos da transmissão vertical na dinâmica da doença.

ALMEIDA, G. C.; SANTOS, F. L. P. Modelo matemático espaço-discreto para análise de propagação da dengue. C. Q. D. - Revista Eletrônica Paulista de Matemática , Bauru, v. 7, p. 78-87, dez. 2016. Ediçao ERMAC

DOI: 10.21167/cqdvol7ermac201623169664gcaflps7887 - Disponível em: http://www.fc.unesp.br/\#!/departamentos/matematica/revista-cqd/ 


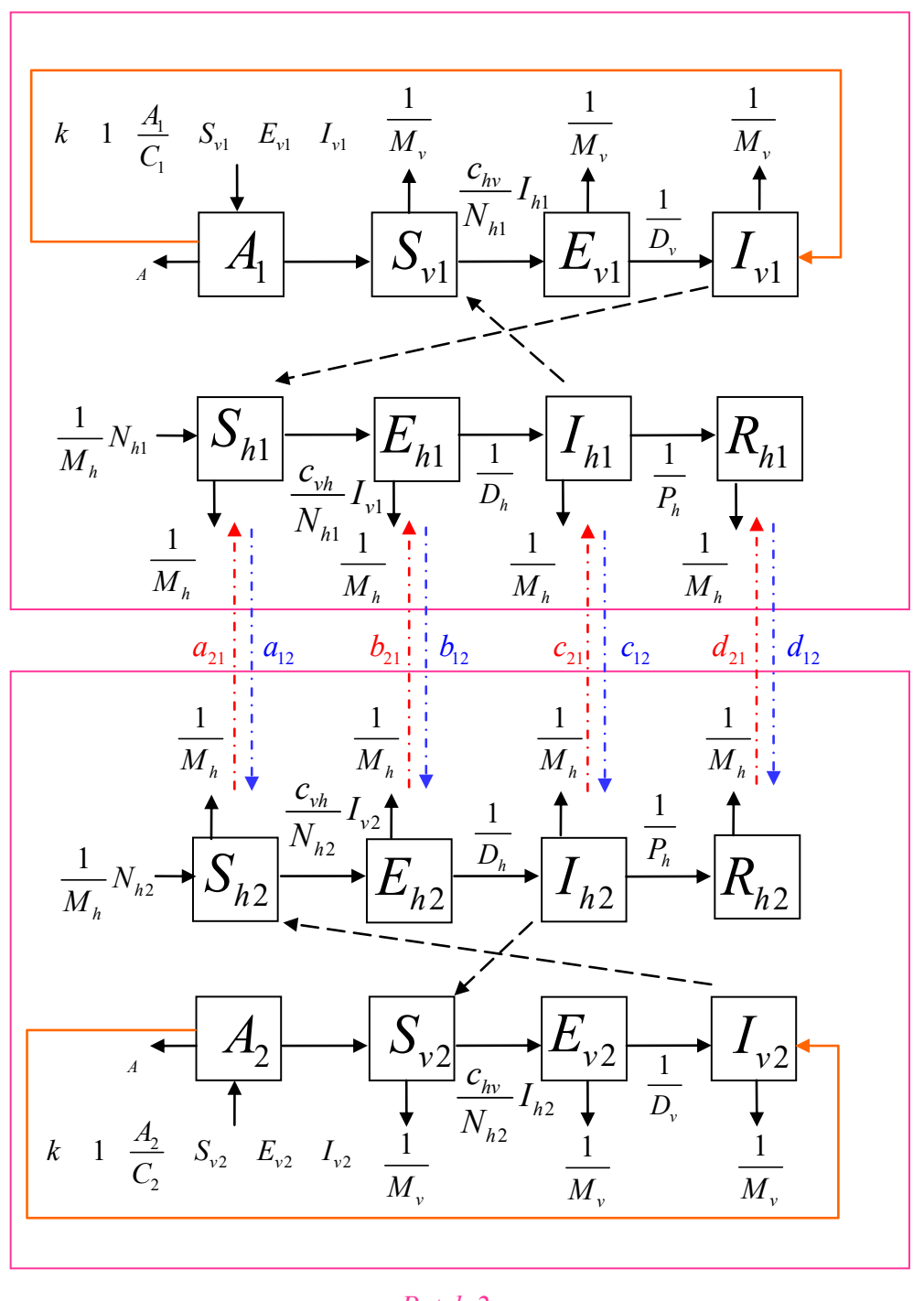

Patch 2

Figura 1: Fluxo entre os dois patches. A movimentação humana entre os dois patches e seus respectivos compartimentos está representado pelas setas pontilhadas nas cores vermelha e azul. A seta contínua na cor laranja denota a transmissão vertical.

\section{Resultados Numéricos}

Consideramos cinco cenários para as simulações numéricas realizadas para o sistema (1), conforme mostra a Tabela 3. Para obtenção da solução numérica do sistema foi utilizado em todas as simulações o método de Runge-Kutta de quarta ordem no intervalo de $[0,1000]$ dias e passo-no-tempo $d t=0.001$.

As simulações foram implementadas em linguagem $\mathrm{C}$ no sistema operacional Linux Mint 17.3 Cinnamon com processador Intel Core i5 e memória de 8 GB. Usando essas configurações o tempo de resolução ficou entre 0.01 a 0.06 segundos. O software aplicado para plotagem dos gráficos foi o Xmgrace. 
Tabela 3: Cenários das Simulações Numéricas.

\begin{tabular}{ccc}
\hline Cenários & Mobilidade Humana & Transmissão Vertical \\
\hline $\mathbf{1}$ & - & - \\
$\mathbf{2}$ & $\checkmark$ & - \\
$\mathbf{3}$ & $S_{h}, E_{h} \mathrm{e} R_{h}$ & - \\
$\mathbf{4}$ & $S_{h} \mathrm{e} R_{h}$ & - \\
$\mathbf{5}$ & - & $\checkmark$ \\
\hline
\end{tabular}

Os resultados numéricos obtidos mostram os efeitos da mobilidade humana e da transmissão vertical no desenvolvimento da dengue no patch que estava inicialmente livre da doença. Os valores dos parâmetros biológicos utilizados estão disponíveis na Tabela 2. As taxas de movimentação humana $a_{i j}, b_{i j}, c_{i j}$ e $d_{i j}$ são tomadas aleatoriamente em $[0,1]$, para todo $i, j=1,2$. As condições iniciais das variáveis de estado para o primeiro patch foram: $A_{1}=1000, S_{h 1}=$ $9950, I_{h 1}=50, S_{v 1}=2000$ e $E_{h 1}=R_{h 1}=E_{v 1}=I_{v 1}=0$, ou seja, esse patch têm condições para a transmissão da doença dengue. Já o segundo patch está livre da doença, com as seguintes condições iniciais: $A_{2}=100, S_{h 2}=10000, S_{v 2}=2000$ e $E_{h 2}=I_{h 2}=R_{h 2}=E_{v 2}=I_{v 2}=0$.
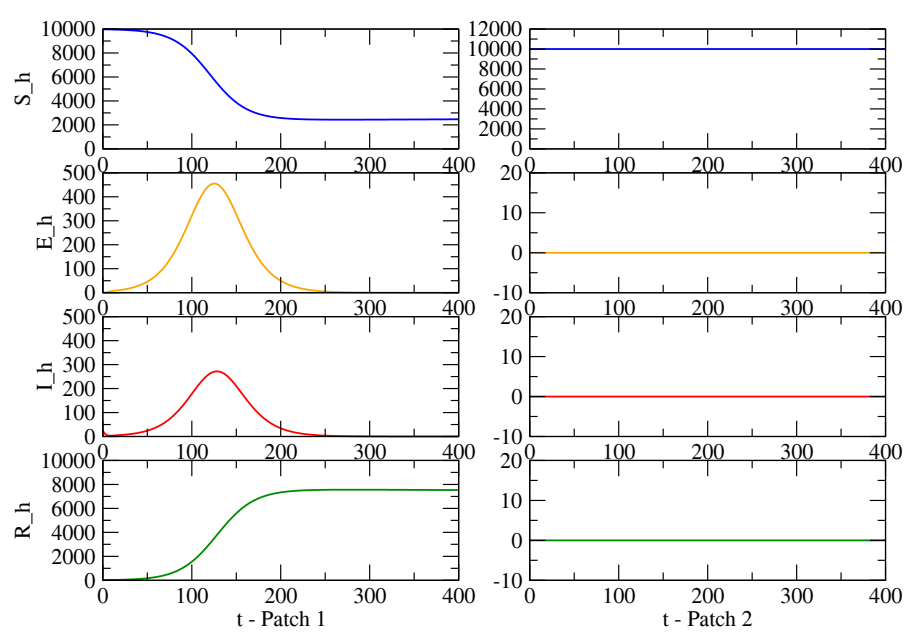

Figura 2: Dinâmica populacional para dois patches com mobilidade nula em todos os compartimentos de humanos usando o parâmetro de simulação $\beta=0$ (transmissão vertical).

No primeiro cenário, temos que não há mobilidade em nenhum dos compartimentos da população humana, ou seja, as taxas de movimentação são todas nulas. Além disso, não consideramos também a transmissão vertical $(\beta=0)$. A Figura 2 exibe os resultados numéricos da simulação do cenário 1 , em que esses mostram a ocorrência da epidemia no primeiro patch, devido a existência de condições para dengue. O segundo continuou livre da infecção, como era de se esperar, devido ausência da mobilidade humana e da transmissão vertical.

No segundo cenário, não consideramos a transmissão vertical $(\beta=0)$ e foi fixado mobilidade em todos os compartimentos de humanos $\left(S_{h}, E_{h}, I_{h}\right.$ e $\left.R_{h}\right)$. Os respectivos resultados numéricos são mostrados na Figura 3, no qual observamos que devido ao fato da mobilidade humana ser considerada neste caso, o patch que não possuía condições para a dengue, passou a ter a epidemia da doença. 

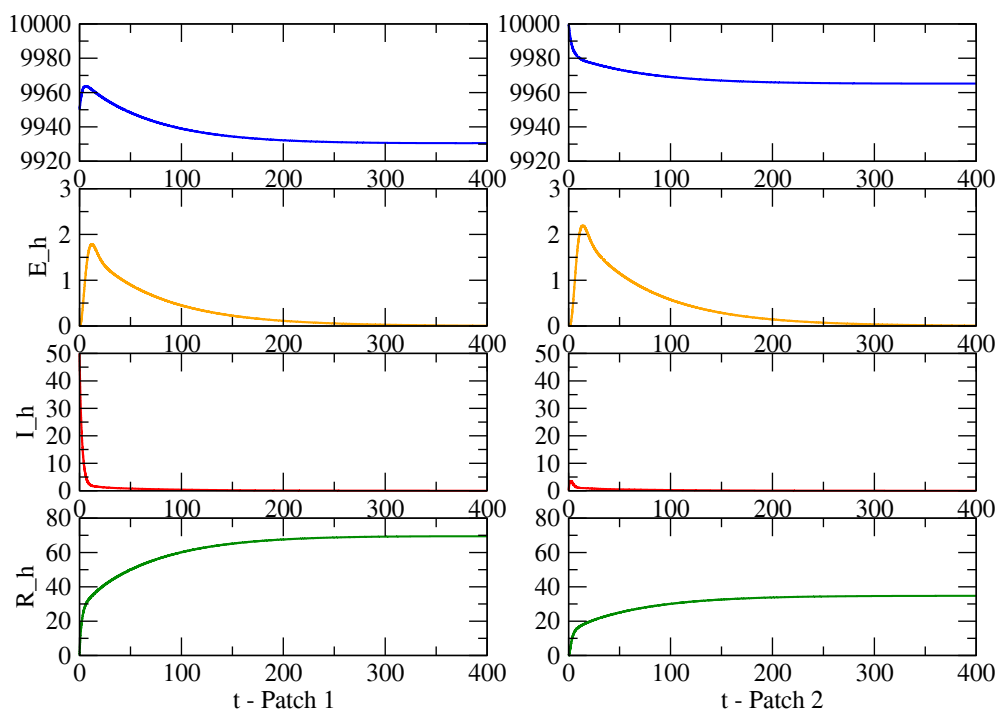

Figura 3: Dinâmica populacional para dois patches sobre os efeitos da migração humana em todos os compartimentos de humanos com o parâmetro de simulação $\beta=0$ (transmissão vertical).
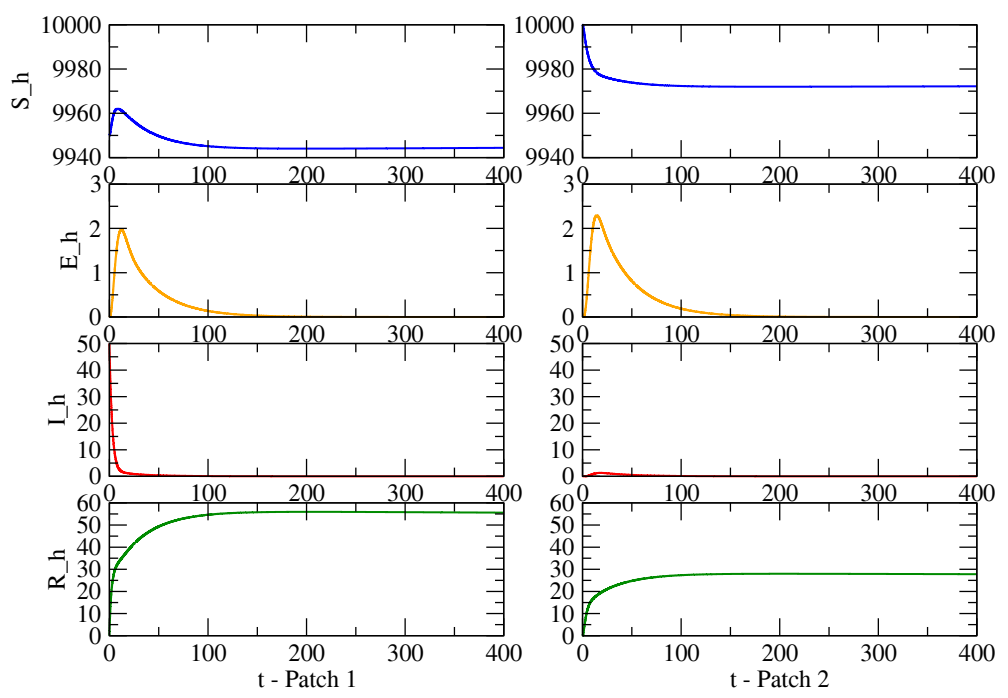

Figura 4: Dinâmica populacional para dois patches sobre o efeito da mobilidade na subpopulação de humanos expostos (aqueles que não apresentam sintomas e transmitem dengue depois de um certo período de tempo) com o parâmetro de simulação $\beta=0$ (transmissão vertical).

No terceiro cenário, a transmissão vertical $(\beta=0)$ não foi imposta e consideramos que humanos infecciosos estão seguindo as recomendações necessárias para uma recuperação total dos sintomas vinculados a dengue, isto é, repouso absoluto. Logo, consideramos mobilidade humana nos demais compartimentos $S_{h}, E_{h}$ e $R_{h}$. Devido a mobilidade de indivíduos expostos (pessoas que estão infectadas e depois de um período começam a transmitir dengue), o segundo patch passou a ter a presença da doença, como podemos observar nos resultados numéricos exibidos na Figura 4.

A Figura 5 exibe os resultados do quarto cenário, cuja transmissão vertical continua nula e existe mobilidade apenas nos compartimentos de humanos susceptíveis e recuperados. Como esperado, o segundo patch continuou sem a doença (condição inicial), pois humanos expostos e infecciosos não saíram do seu patch atual.

ALMEIDA, G. C.; SANTOS, F. L. P. Modelo matemático espaço-discreto para análise de propagação da dengue. C. Q. D. - Revista Eletrônica Paulista de Matemática , Bauru, v. 7, p. 78-87, dez. 2016. Ediçao ERMAC

DOI: 10.21167/cqdvol7ermac201623169664gcaflps7887 - Disponível em: http://www.fc.unesp.br/\#!/departamentos/matematica/revista-cqd/ 

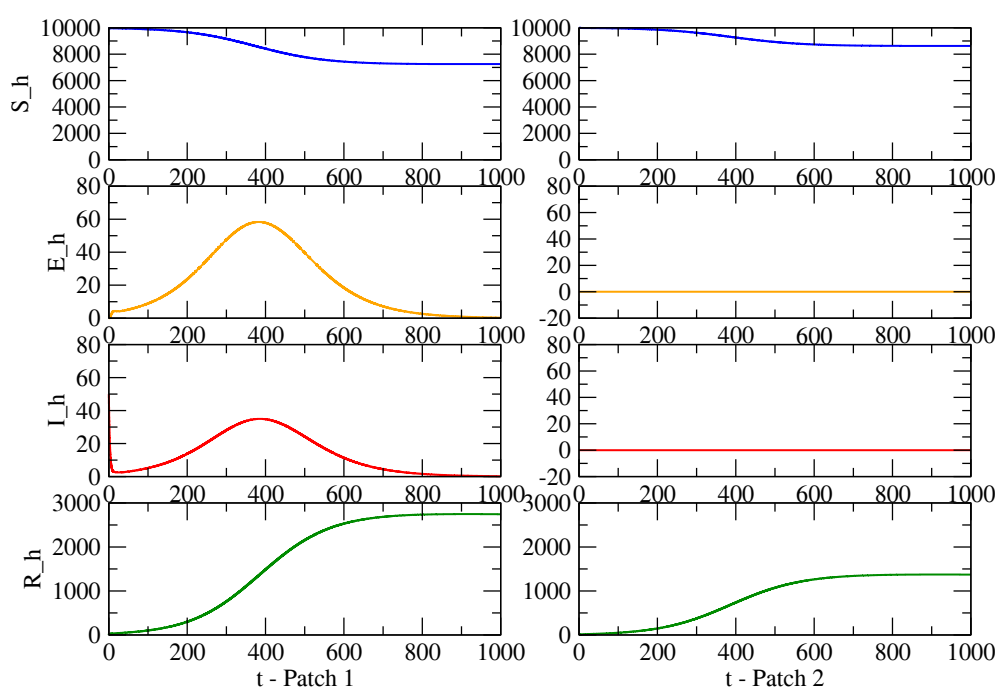

Figura 5: Dinâmica populacional para dois patches sobre o efeito da mobilidade nas subpopulações de humanos suscetíveis e recuperados em que o parâmetro de simulação $\beta=0$ (transmissão vertical).

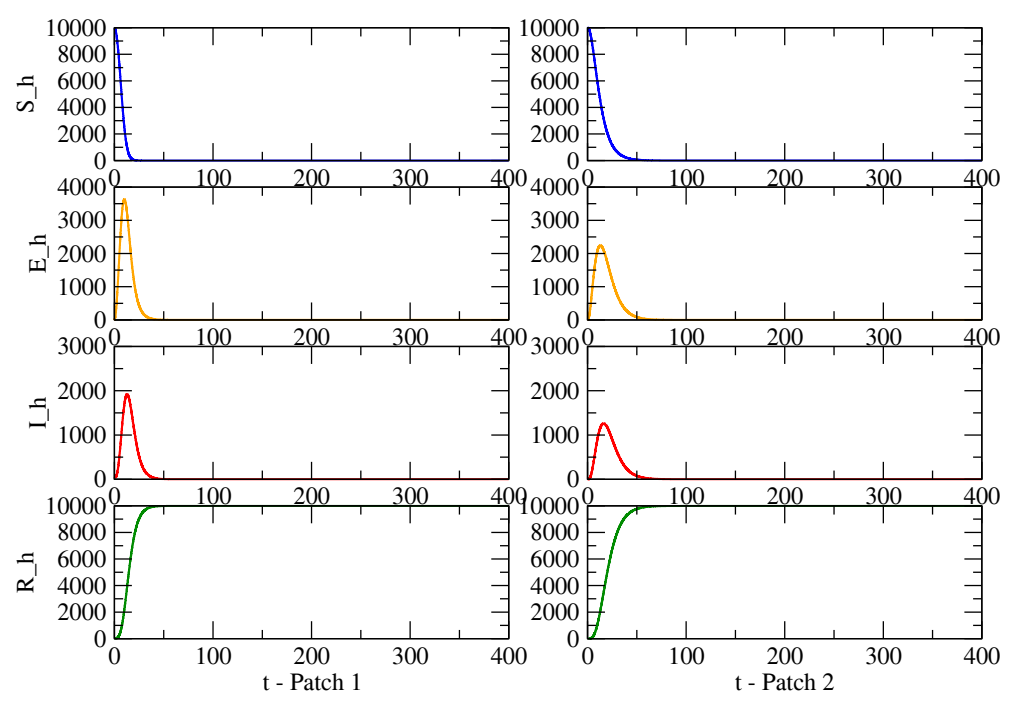

Figura 6: Dinâmica populacional para dois patches sobre os efeitos da transmissão vertical para $\beta=0.3$.

Por fim, a Figura 6 exibe os resultados da simulação do quinto cenário, que não possui mobilidade humana em nenhum compartimento, mas consideramos neste processo a transmissão vertical (transovariana ou transovo) com uma proporção de $30 \%$ do total da oviposição dos mosquitos fêmeas. Portanto, observamos que a epidemia da doença dengue surgiu no segundo patch com uma maior influência do que a intervenção da mobilidade humana, ou seja, a epidemia foi mais intensa, surgindo em um curto espaço de tempo com relação aos demais cenários.

As principais conclusões acerca da simulação numérica da dinâmica da dengue em patches são sumarizadas na seção seguinte. 


\section{Conclusão}

Para verificar a dinâmica populacional do modelo proposto e o papel da transmissão vertical, simulações numéricas foram realizadas para o caso de dois patches, sendo um com condições iniciais para a enfermidade (população de humanos infecciosos) e o outro livre da doença (somente a subpopulação de humanos e mosquitos susceptíveis).

Os resultados numéricos obtidos mostram os efeitos da mobilidade humana já esperados para o desenvolvimento da dengue no patch que estava livre inicialmente da doença e da importância da transmissão vertical como requisitos para a introdução do vírus em áreas livres da dengue.

Além disso, a transmissão vertical do mosquito da dengue ainda não é uma discussão considerada clara, mas acredita-se que seja umas das principais formas de permanência do vírus nas populações de vetores, surgindo assim as epidemias nas populações de humanos e na durabilidade do vírus no decorrer dos anos.

Conforme essas importantes apurações, podemos sugerir o reforço das ações de controle na fase aquática para o ciclo do vírus da dengue, como forma de conter a doença e minimizar esse grande problema da saúde pública mundial, uma vez que não é possível frear a mobilidade dos seres humanos devido suas atividades diárias.

\section{Referências Bibliográficas}

ANDRAUD, M.; et al. Dynamic epidemiological models for dengue transmission: a systematic review of structural approaches, PLoS ONE, v. 7, n. 11, 2012. Disponível em:

$<$ http://journals.plos.org/plosone/article?id=10.1371/journal.pone.0049085>.

Acesso em: 01 fev. 2016.

ARINO, J.; VAN DEN DRIESSCHE, P. A multi-city epidemic model. Mathematical Population Studied, v. 10, n. 3, p. 175-193, 2003.

BASSANEZI, R. C. Equações diferenciais ordinárias: Um Curso Introdutório. São Paulo: UFBAC-CMCC, 2011. v. 1. Disponível em:

$<$ http://gradmat.ufabc.edu.br/disciplinas/listas/iedo/notasdeaulas/equacoes-diferenciais-

ordinarias-rodney.pdf $>$. Acesso em: 30 mai. 2016.

BOWONG, S.; DUMONT, Y.; TEWA, J. J. A patchy model for chikungunya-like diseases. Biomath, v. 2, n. 1, p. 1-19, 2013. Disponível em:

$<$ http://www.biomathforum.org/biomath/index.php/biomath/article/view/j.biomath.2013.07.237/ 279>. Acesso em: 03 mar. 2016.

CIRINO, S.; SILVA, J. A. L. Modelo epidemiológico SEIR de transmissão da dengue em redes de populaç oes acopladas. Revista TEMA - Tendências em Matemática Aplicada e Computacional, Porto Alegre, v. 5, n. 1, p. 55-64, 2004.

DENGUE: guidelines for diagnosis, treatment, prevention and control. New ed. Geneva: World Health Organization, 2009.

ALMEIDA, G. C.; SANTOS, F. L. P. Modelo matemático espaço-discreto para análise de propagação da dengue. C. Q. D. - Revista Eletrônica Paulista de Matemática , Bauru, v. 7, p. 78-87, dez. 2016. Ediçao ERMAC.

DOI: 10.21167/cqdvol7ermac201623169664gcaflps7887 - Disponível em: http://www.fc.unesp.br/\#!/departamentos/matematica/revista-cqd/ 
ESTEVA, L.; VARGAS, C. Analysis of a Dengue Disease Transmission Model. Mathematical Biosciences, v. 150, n. 2, p. 131-151, 1998.

ESTEVA, L.; YANG, H. M. Assessing the effects of temperature and dengue virus load on dengue transmission. Journal of Biological Systems, v. 23, n. 4, p. 527-554, 2015.

GENERALI, D. T. Sincronização de metapopulações heterogêneas. 2010. 83 f. Dissertação (Mestrado em Matemática Aplicada) - Universidade Federal do Rio Grande do Sul, Porto Alegre, 2010.

GERHARDT, M. P. Modelo de transmissão da dengue com competição larval uniforme. 2004. 112 f. Dissertação (Mestrado em Matemática Aplicada) - Universidade Federal do Rio Grande do Sul, Porto Alegre, 2004.

KERMACK, W. O.; MCKENDRICK, A. G. A contributions to the mathematical theory of epidemics. Proceedings of the Royal Society A: mathematical, physical, and engineering, v. 115, n. 772, p. 700-721, 1927. Disponível em:

$<$ http://rspa.royalsocietypublishing.org/content/royprsa/115/772/700.f ull.pdf $>$. Acesso em:

14 jan. 2016.

LEANDRO, D. C. Transmissão transovariana no vírus da dengue soropositivo 2 em Aedes Aegypti (Diptera: culicidae) e suas implicações na biologia reprodutiva do mosquito. 2015. 133 f. Tese (Doutorado em Zoologia)- Universidade Federal de Pernambuco, Recife, 2015.

NEWTON, E. A. C.; REITER, P. A model of the transmission of dengue fever with an evaluation of the impact of ultra-low volume (ULV) insecticide applications on dengue epidemics. The American Society of Tropical Medicine and Hygiene, v. 47, n. 6, p. 709-720, 1992.

PUANGSUN, S.; PATANARAPEELERT, K. An SIR epidemic model with gravity in patchy environment: analyses for two patches system. KMITL Science and Technology Journal, v.12, n.2, p.127-133, 2012.

SANTOS, F. L. P. A general discrete patches approach to investigate the populational dynamics of mosquitoes and humans in dengue. Proceeding Series of the Brazilian Society of Computational and Applied Mathematics, v. 3, n. 2. Vitória-ES: SBMAC, 2015. p. 1-7.

STOLERMAN, L. M.; COOMBS, D.; BOATTO. SIR-network model and its application to dengue fever. SIAM J. Appl. Math., v. 75, n. 6, p.2581-2609, 2015.

THOMÉ, R. C. A.; YANG, H. M.; ESTEVA, L. Optimal control of Aedes aegypti mosquitoes by the sterile insect technique and insecticide. Mathematical Biosciences, v. 223, n. 1, p. 12-23, 2010 . 